\title{
Letter to the Editor concerning "The incidence of myocardial infarction after lumbar spine surgery" by Harwin B, Formanek B, Spoonamore M, Robertson D, Buser Z, Wang JC (Eur Spine J. 2019; doi:10.1007/s00586-019-06072-4)
}

\author{
Teruhiko Imamura ${ }^{1}[$
}

Received: 24 July 2019 / Revised: 24 July 2019 / Accepted: 17 August 2019 / Published online: 22 August 2019

(c) Springer-Verlag GmbH Germany, part of Springer Nature 2019

To the Editor,

I read with great interest a manuscript written by Harwin and colleagues, demonstrating that the preoperative risk factors, patient demographics, and surgical procedure type were associated with an increased incidence of myocardial infarction following lumbar spine surgery using a large-scale cohort. We believe that their findings would contribute to preoperative risk stratification and improved perioperative management. I have several concerns to improve the implication of their findings.

They stratified the incidences of postoperative myocardial infarction by perioperative variables and found several risk factors including age, male gender, fusion surgery, and congestive heart failure. However, they did not consider confounders among them. Multivariate regression analyses might be useful to find independent risk factors. Furthermore, considering their risk ratio, novel risk scoring system as a summation of the weighted scores of each risk factor can be constructed. Predictability of the novel scoring system will be compared to the conventional Charlson comorbidities index score that they used.
Next concern is whether an aggressive intervention to each preoperative risk factor would reduce the incidence of postoperative myocardial infarction. Given the highest risk ratio, congestive heart failure might be a major therapeutic target. Recently developed vasopressin type 2 receptor antagonist, tolvaptan, might be a useful therapeutic tool to improve patients' congestion maintaining hemodynamics, given hypertension and hypotension are other major risk factors.

\section{Compliance with ethical standards}

Conflict of interest None of the authors has any potential conflict of interest.

Publisher's Note Springer Nature remains neutral with regard to jurisdictional claims in published maps and institutional affiliations.
Teruhiko Imamura

te.imamu@gmail.com

1 Division of Cardiology, Department of Medicine, University of Chicago Medical Center, 5841 S Maryland Avenue,

Chicago, IL 60637, USA 